\title{
Advanced Android Application For Law And Order Using Data Mining
}

\author{
Sanal Sajeevan', Ajil T U², Fahad P J $\mathbf{J}^{3}$, Deepak K N ${ }^{4}$ \\ Student, Department of Computer Science Engineering, Universal Engineering College, Vallivattom ${ }^{1,2,3}$ \\ Assistant Professor, Department of Computer Science, Universal Engineering College, Vallivattom ${ }^{4}$
}

\begin{abstract}
The Indian Penal Code (IPC) is the main criminal code of India. It is a comprehensive code intended to cover all substantive aspects of criminal law. Majority of people are unaware of the legal systems prevailing in the country and also of their constitutional rights. Even though people are aware of it, they are not in a situation to afford because of their economic and social backwardness. They are in a helpless situation to engage the services of legal counsel, which has become a costly affair. This project intends to overcome all these problems. Through this we can improves the people's awareness about the Indian law's as well as this system act as a mediator between the clients and the lawyers. This also provides online support during the emergency situations like police checking, petty cases, warrant issue, etc. People and lawyer can add quotes as well as anyone can react to the quotes. Legal documents and judgments (past case histories) from different courts are available for the lawyers which would be helpful for lawyers for practicing and this facility is implemented using data mining technology.
\end{abstract}

Keywords: Data Mining, Information Extraction, Data Base Searching, Video Call

\section{INTRODUCTION}

The Indian Penal Code (IPC) is the main criminal code of India. It is a comprehensive code intended to cover all substantive aspects of criminal law. Majority of people are unaware of the legal systems prevailing in the country and also of their constitutional rights. Even though people are aware of it, they are not in a situation to afford because of their economic and social backwardness. They are in a helpless situation to engage the services of legal counsel, which has become a costly affair.

This project intends to overcome all these problems. Through this we can improves the people's awareness about the Indian law's as well as this system act as a mediator between the clients and the lawyers. This also provides online support during the emergency situations like police checking, petty cases, warrant issue, etc. People and lawyer can add quotes as well as anyone can react to the quotes. Legal documents and judgments (past case histories) from different courts are available for the lawyers which would be helpful for lawyers for practicing and this facility is implemented using data mining technology.

\section{RELATED WORKS}

Legal case management software programs are designed to assist lawyers or law firms manage their cases including client records, schedules and appointments, deadlines, billing and bookkeeping, computer files, and documents. It also facilitates compliance to policies and requirements laid down by judicial and legal authorities.

In "Indian Laws", although the lawyers are studying all about the laws and codes in their law schools, it's literally impossible to remember all the laws in our minds. For those who struggle to remember all of the thousands of laws and recall them when it's needed, the Indian Laws app is the solution.

The next application is "ScheduLAWyer", just like the Lawyer Diary app, this app will work as your work diary. With this app loaded into your Android device, you can stay organized with your appointment schedules, dates, as well as with your case-related information.

In "IPC - Indian Penal Code", as the name suggests, this android application is all about Indian Penal Codes. The latest version of the application is giving the user with a good source of reference to Indian lawyers, as well as law students. There are sections and sub-sections within each chapter to make your search process easier. What makes this the best app is its simple user interface and readable text.

In $^{[1]}$ Query Reorganisation Algorithms for Efficient Boolean Information Filtering, In recent years, information filtering (IF) applications (also known as information dissemination or publish/subscribe), such as news alerts, weather monitoring, and stock quotes, have gained popularity. Such applications assist users to cope with the information avalanche and the cognitive overload associated with it. For the case of news alerts, digital libraries, or RSS feeds, where the data of interest is mostly textual, users express their needs using information retrieval languages and submit 


\title{
International Journal of Advanced Research in Computer and Communication Engineering
}

\author{
Vol. 8, Issue 3, March 2019
}

continuous queries (or profiles) to a server, thus, subscribing to newly appearing documents that will satisfy the query conditions. The server will then be responsible for notifying the subscribed users automatically whenever a new document that matches their information needs is published. Publishers can be news feeds, digital libraries, or even users who post new items to blogs, social media, and Internet communities. This functionality is very different from Information Retrieval (IR) applications like search engines. Specifically, in IR when a query is posed, a single search is executed and the current matching data items are presented to the user. Contrary, in IF the server indexes the user queries rather than the data and evaluates newly published data items against the stored continuous queries. In more detail, the problem of information filtering may be defined as follows: given a database DB of continuous queries that reside on a server and an incoming document $d$, retrieve all queries q2DB that match $d$. The filtering problem is of high importance and needs to be solved efficiently, since servers are expected to handle millions of user queries and high rates of published documents. Efficiency issues were identified by many researchers that proposed tree and trie-based algorithms for supporting fast filtering under various data models (e.g., flat attribute-based, semi-structured XML) and query languages (e.g., Boolean, VSM), both for main-memory and secondary storage. However, all these approaches use a greedy clustering method that is sensitive to the insertion order of submitted queries and do not consider that an evolving query workload might require the reorganisation of the query database to achieve efficient filtering performance.

In this work, we concentrate on textual IF and present a novel trie-based, main-memory algorithm for Boolean IF that is able to match incoming documents against millions of queries in a few milliseconds. Our method uses linguistically motivated concepts, such as words, to support continuous queries that are comprised of conjunctions of keywords and may be used as a basis for query languages that support not only basic Boolean operators, but also more complex constructs, such as proximity operators and attributes. We believe that offering an efficient Boolean filtering service is a valuable addition to any text filtering setup. Boolean IR/IF is still the model of choice of advanced users that want total control of their results and is widely supported in systems of major stakeholders like Google's advanced search/alert mechanisms, Oracle's text extender module, and in Apache's text search engine. Such systems, that are meant to cope with a high workload and are designed for efficiency, are possible applications for our work. Our algorithm, coined STAR (acronym for STAtistical Reorganisation), is the first in the literature to consider database reorganisation (through appropriate word/query statistics) to achieve efficient textual IF under the Boolean model. The main idea behind the proposed algorithm is to use tries to capture common elements of queries. However, the key differences with these approaches lie in the collection and utilisation of statistics on the importance of keywords in the indexed queries, the reorganisation of the query database according both to word and query importance, and the demonstration that the nature of the trie forest is more important than its compactness when it comes to filtering efficiency. Interestingly, all previous works were aiming at minimising the size of the trie forest, since there was an implicit conjecture that a small forest would result in lower filtering times due to less node visits.

In this work, we demonstrate that forest size is not the dominating optimisation factor when it comes to filtering efficiency; contrary, the focus should be put on the nature of the tries and on qualitative characteristics (expressed through heuristics).

$\mathrm{In}^{[2]}$ People tend to pay bribes to get their work done or get rid of any issue. People are unaware of the existing laws because of which they are defrauded. The illiteracy about laws has become advantageous to the deceiver. Hence, proper education should be given to the people regarding the laws of the Indian Constitution. The mobile application serves the purpose of providing valuable information about laws, based on the question posted by the user. An information retrieval system is designed to retrieve relevant answers about laws. The keywords from laws of Indian Constitution are indexed and used to build a store of indexed keywords. The user query is processed using natural language processing. Referring with the indexed keywords, multiple laws are identified. The laws are ranked in line with the reward gained. The system is further modeled to master based on the positive and negative feedback from the user. The reward is revised in accordance with the feedback. Thus, the system learns from the user too. Considering everything, the system is designed to deliver possible solution based on the user feedback. Overall, People tend to pay bribes to get their work done or get the system solves the problem of lack of awareness about laws of the Indian constitution.

$\mathrm{In}^{[3]}$ Android is a mobile Operating System (OS) based on the Linux kernel and currently developed by Google. With a user interface based on direct manipulation, Android is designed primarily for touchscreen mobile devices such as smart phones and tablet computers. Android's source code is released by Google under open source licenses, although most Android devices ultimately ship with a combination of open source and proprietary software,including proprietary software developed and licensed by Google. Android is popular with technology companies which require a ready-made, low-cost and customizable operating system for high-tech devices. Android's open nature has encouraged a large community of developers and enthusiasts to use the open-source code as a foundation for community-driven projects, which add new features for advanced users or bring Android to devices which were officially released running other operating systems.

$\mathrm{In}^{[4]}$ Connectify application is an integration of social networking and E-commerce. It majorly focuses on the freelancer business growth and their development where the fresh faces in the world of business can sell their talent in 
the form of art, dance, singing, photography, etc to the relevant customer population by the means of our application and online transactional procedure. Connectify networks is build on PHP MySQL database using android studio for the structural and design part of the main application. The content from users they have subscribed to will be visible in the subscribed tab and categories they have subscribed to will be visible in the feed tab. Companies are increasingly using online communities to create value for the firm and their customers. Ensuring that brand activity is relevant to a social network's core audience is crucial for advertisers wanting to tap into niche communities It is a concept derived from the drawbacks of different application with the motive to combine their respective function. This Application will serve them as a good place to share good work and a place where their work will be appreciated by the people.

$\mathrm{In}^{[5]}$ The amount of crime in our country have been raised and most of the people are unaware of the criminal laws under IPC (Indian Penal Code). Therefore it becomes necessary to make the citizens aware of all kinds of laws put forth by our constitution against crime so that people come forward to register case against it. In admin login, the admin add the laws of IPC and their respective crimes. Those laws are categorized into sections. There is also search option available where user can find or search according to requirement by entering the query. In user login, the user can search for the laws based on the crimes. The user can view both the crime details and law details. It also provides lawyer details such as name of lawyer and location. This helps the user to know the crime and respective laws. The user can send queries related to laws and admin can view those queries and reply. The project " ANDROID LAW SYSTEM" is designed using Standard Android 4.0.3 platform. The platform used to develop the application is Eclipse IDE (Mars) with Java 1.6 Standard Edition.

$\mathrm{In}^{[6]}$ Nowadays, technology is increasingly used by human being in every field. As people move from one place to another, many wireless technologies are available to remain in contact with others, without regard of the location. The increasing popularity of Smart Phones has drawn the attention of almost everybody. Along with making and receiving calls, users can send and receive messages, access the Internet, digital media, incorporate audio/video recording etc. Smart Phones also contain built-in keyboard, high resolution camera, front side camera for video conferencing, touch screen etc. Different smart phones have different operating systems. A mobile app, short for mobile application or just app is an application which runs on smart phones, tablet or mobile phones. Apps are pre installed or downloadable pieces of software that can do almost everything. Apps make mobile more like portable computers having multi core processors, gigabytes of memory and a real operating system. Originally mobile apps are made available for informational purposes that include Gmail, calendar, weather information etc. With increase in technology and user demands, developers started to make apps for other purposes like games, banking, video chats etc. An app can show the data in a similar way as a website, along with other benefits to download the content that can be used offline, in case the Internet is not available. There are many apps available in market today for different Operating Systems i.e. Android, Blackberry and Apple etc., in which Android is having the maximum market share these days. Figure depicts market share of different operating systems from 2011 to 2014.

$\mathrm{In}^{[7]}$ Today the world is full of technologies; there are lots of inventions made to use the latest technologies. To be specific on technology, Mobile phones have been a part of our everyday use where we share lots of information through this communication channel. Now a day's android system is becoming more popular, especially in the Smartphone market. Because there are some of the development tools which is available free are used to generate many applications in an android platform. The main reason for its popularity is, it also provides a very convenient hardware platform for developers. In android, there are applications for SMS controller, SMS spam and Auto-reply features. Some of the android applications allow creating group between the family and friends to share their messages and location. Group scheduling feature is also implemented in some android application to improve the response time. In this paper section 2 explain the related works of this application. Section 3 explain the concept of the application relates to the implementation such as system architecture, modules, functions to develop this application and its working principles. Section 4 conclude the overview of the application along with the future enhancements.

$\mathrm{In}^{[8]}$ Cloud based instant messaging system for android smartphone with help of internet is specially design for the people to communicate with their families, friends, colleague.in any organization employees can send and receive messages virtually without face to face contacting; meanwhile the employees can share data and information through instant messaging application. What's more, the staff can talk to customers or vendors online as well, in other word, now people can do business through the instant messenger direct rather than use the traditional method like make phone calls. The use of instant messaging nowadays is more than the calling function itself. This paper puts forward the design method of instant messaging system based on SOAP and cloud computing technology for android Smartphone. SOAP interface is an application programming interface of TCP/IP network. Cloud platform is a platform where Cloud Application runs, it is an online application, which works over internet. Cloud Platform has been constructed of three layers: "SaaS", "PaaS", and "Iaas" . In this research work, cloud platform is used for deploying database of instant messaging application.

$\mathrm{In}^{[9]}$ Electronic payment system is a mode of payments over an electronic network such as the internet. In other words we can say that e-payment is a method in which a person can make Online Payments for his purchase of goods and services without physical transfer of cash and cheques, irrespective of time and location. Electronic payment 


\title{
International Journal of Advanced Research in Computer and Communication Engineering
}

\author{
Vol. 8, Issue 3, March 2019
}

system is the basis of on-line payments and on-line payment system development is a higher form of electronic payments. It makes electronic payments at any time through the internet directly to manage the e-business environment.

$\mathrm{In}^{[10]}$ The mobile payment market is currently under transition with a history of numerous tried and failed solutions and a future of promising but yet uncertain possibilities with contactless RFID and other new potential technologies. At this point of the development we take a look at the current state of the mobile payment market, review prior literature on mobile payment services, analyze the different factors that impact the market, and give directions for future research on this still emerging field. To facilitate the analysis, we propose a framework of four contingency and five competitive factors, and organize the contemporary mobile payment research under the proposed framework.

$\mathrm{In}^{[11]}$ Application framework defined the common structure of programs in the specific domain. Essentially, a framework is a component that can be reused, it set the architecture of applications and incorporated as a set of abstract classes and the cooperation of their instances. Android is an open source operating system based on Linux kernel and launched by Google. Unlike PC operating system, mobile phone operating systems are constrained by their hardware, storage space, power dissipation and mobility conditions. Compared with the development of applications on PC, there are some different features of applications on mobile phone operating systems. This paper introduced the basic architecture and application framework of Android operating system, gives a detailed description of main structure of Android applications and the methods of developing applications based on Android application framework.

$\mathrm{In}^{[12]}$ With the era of network economy, e-commerce is rapidly emerging as the latest mode of commodity trading. Electronic payment, as an intermediary link, is the most concerned issue for both sides in e-commerce transaction. Electronic payments refers to the parties in electronic transactions, including consumers, manufacturers and financial institutions, taking electronic equipment and various types of trading cards as a medium, computer technology and communication technology as a means and issuing payment instructions directly or indirectly through the network to achieve currency payment and the transfer of funds. With the rapid development of network and change of the payment concept, more and more people begin to use electronic payments. At present in China, the main methods of electronic payment are through online payment via Internet network, mobile payment via communication networks and phone payment etc. In 2006, online payment takes $97 \%$ share in the whole e-payment market. Online payment is still the absolute main form of electronic payment. The number of online store, using the online payment services, has been more than 100,000 at present in China.

$\mathrm{In}^{[13]}$ Information technology and payment systems have witnessed the introduction, acceptance and wide scale deployment of electronic payment systems. The payment system ecosystem has now witnessed the introduction of mobile payment systems and their associated services. Major actors involved in mobile payment systems include telecom operators, banks, merchants and consumers. They need to aggregate their resources and develop a coherent ecosystem which would help the individual actors while also benefiting the overall mobile payment ecosystem. Financial institutions and mobile carriers are becoming increasingly interested and have started collaborating in order to provide mobile payment capabilities which would pave the way for the migration of payment systems from cardbased to phone-based. In a developing country like India, mobile payment systems have experienced rapid growth, deployment and acceptance in a very short span of time. However, these systems are still far from mature and need to be customized and refined further in order to replace or equal the deployment and acceptance of electronic payment systems. Mobile payment services are primarily centred on the unbanked population but also consider the existing population with active bank accounts especially in developing countries.

\section{DESIGN METHODOLOGY}

\section{A. SYSTEM FLOWCHART}

We provide an online interaction for ordinary people with lawyers in emergency situation through this we can improves the people's awareness about the Indian law's as well as this system act as a mediator between the clients and the lawyers. This also provides online support during the emergency situations like police checking, petty cases, warrant issue, etc. People and lawyer can add quotes as well as anyone can react to the quotes. Legal documents and judgments (past case histories) from different courts are available for the lawyers which would be helpful for lawyers for practicing and this facility is implemented using data mining technology. Mobile application serves the purpose of providing valuable information about laws, based on the question posted by the user. An information retrieval system is designed to retrieve relevant information about the laws. The flowchart of the system is shown in Fig. 1 


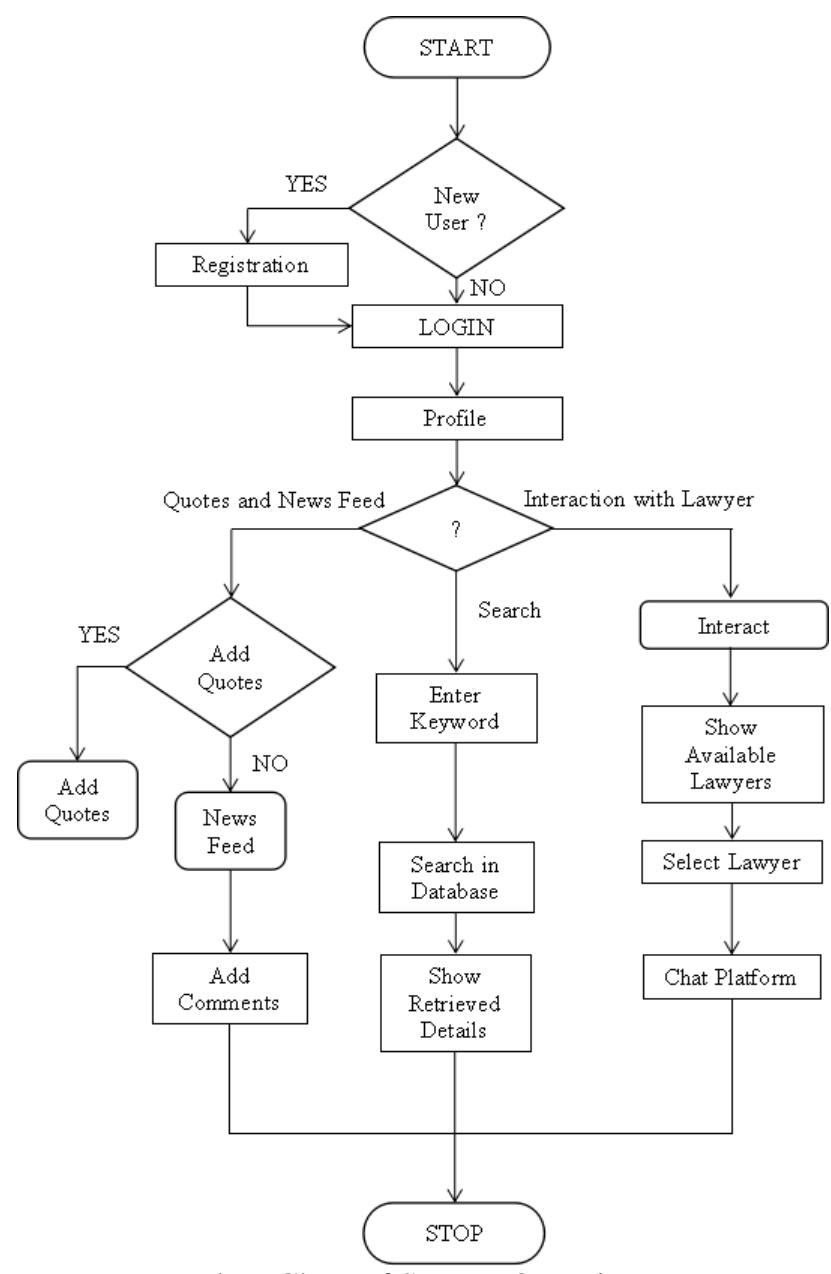

Flow Chart of System Overview

B. BLOCK DIAGRAM

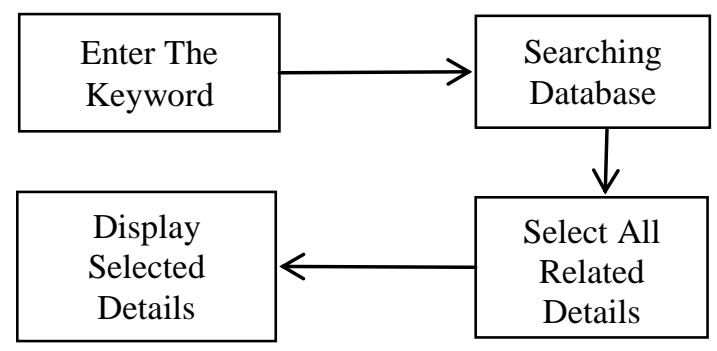

Flow Chart of Data mining

C. SEQUENCE DIAGRAM

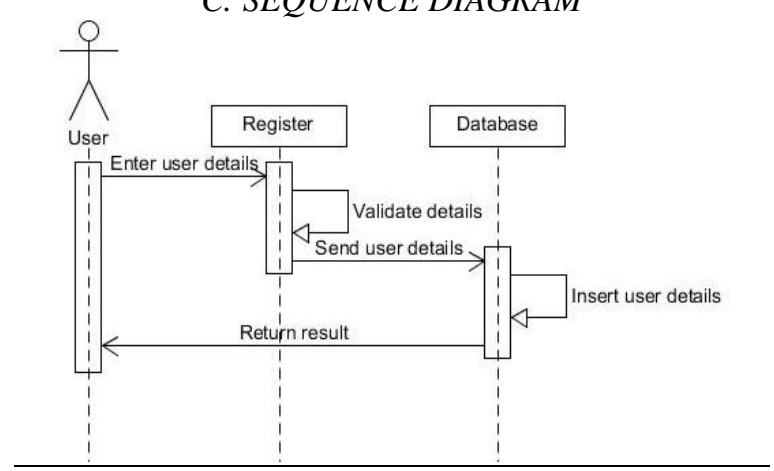

Fig. 3 Sequence diagram of user registration. 
Vol. 8, Issue 3, March 2019

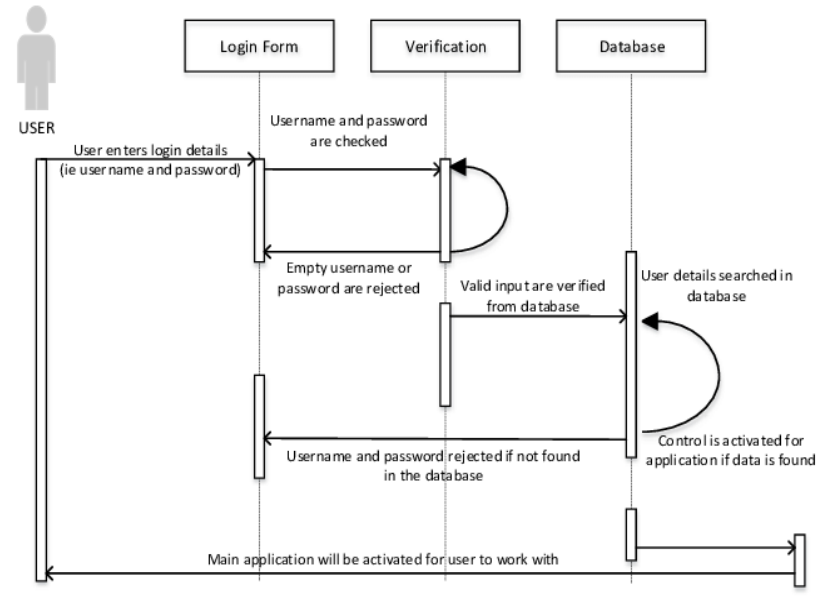

Fig. 4 Sequence diagram of user login.

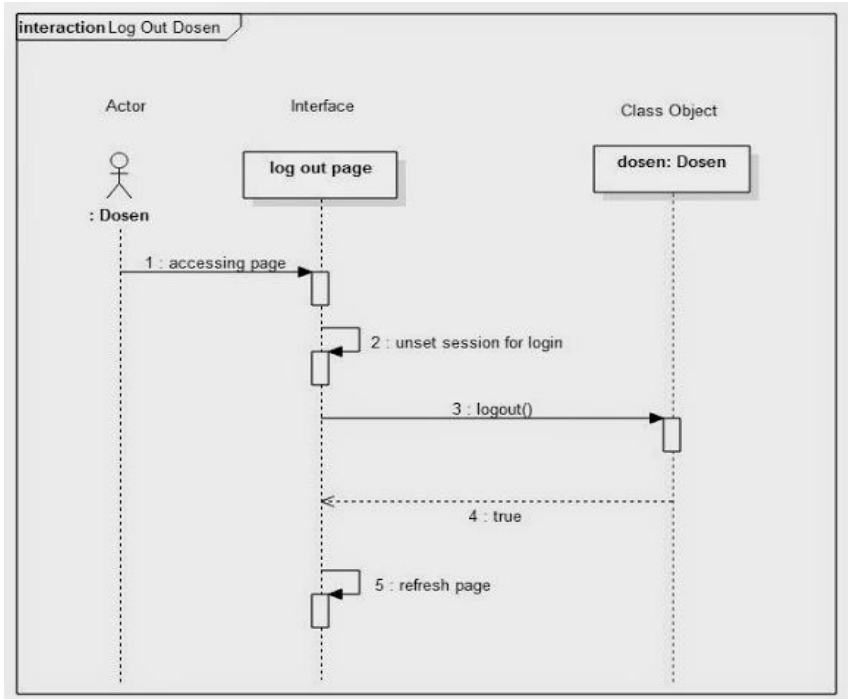

Fig. 5 Sequence diagram of user logout.

\section{SYSTEM OVERVIEW}

\section{A. ANDROID STUDIO}

Android Studio is the official Integrated Development Environment (IDE) for Google's Android operating system, built on JetBrains' IntelliJ IDEA software and designed specifically for Android development. It is available for download on Windows, macOS and Linux based operating systems.It is a replacement for the Eclipse Android Development Tools (ADT) as the primary IDE for native Android application development.

Android Studio was announced on May 16, 2013 at the Google I/O conference. It was in early access preview stage starting from version 0.1 in May 2013, then entered beta stage starting from version 0.8 which was released in June 2014. The first stable build was released in December 2014, starting from version 1.0. The current stable version is 3.3 , which was released in January 2019.

\section{B. MYSQL}

MySQL is an open source relational database management system (RDBMS).Its name is a combination of "My", the name of co-founder Michael Widenius's daughter, and "SQL", the abbreviation for Structured Query Language. MySQL is free and open-source software under the terms of the GNU General Public License, and is also available under a variety of proprietary licenses.

MySQL was owned and sponsored by the Swedish company MySQL AB, which was bought by Sun Microsystems (now Oracle Corporation).In 2010, when Oracle acquired Sun, Widenius forked the open-source MySQL project to create MariaDB. MySQL is a component of the LAMP web application software stack (and others), which is an acronym for Linux, Apache, MySQL,Perl/PHP/Python. 


\title{
International Journal of Advanced Research in Computer and Communication Engineering
}

\author{
Vol. 8, Issue 3, March 2019
}

MySQL is used by many database-driven web applications, including Drupal, Joomla, phpBB, and WordPress. MySQL is also used by many popular websites, including Google (though not for searches), Facebook, Twitter, Flickr and YouTube.

\section{C. $X M L$}

Extensible Markup Language (XML) is a markup language that defines a set of rules for encoding documents in a format that is both human-readable and machine-readable. The W3C's XML 1.0 Specification and several other related specifications - all of them free open standards - define XML. The design goals of XML emphasize simplicity, generality, and usability across the Internet.It is a textual data format with strong support via Unicode for different human languages. Although the design of XML focuses on documents, the language is widely used for the representation of arbitrary data structuressuch as those used in web services. Several schema systems exist to aid in the definition of XML-based languages, while programmers have developed many application programming interfaces (APIs) to aid the processing of XML data.

\section{RESULT}

There were many types of law based apps and software's available in the online markets. Searching about the laws, searching previous case details, new case registrations, searching about lawyer, are the main features now available through these applications. But currently there is no applications which consist of all these features together. If a user need to access these features, user needs to download all these applications separately. It's a disadvantage in the existing system and the unavailability of an online interaction platform between lawyers and clients is also the disadvantage of the existing systems. This also provides online support during the emergency situations like police checking, petty cases, warrant issue, etc. Legal documents and judgments (past case histories) from different courts are available for the lawyers which would be helpful for lawyers for practicing and this facility is implemented using data mining technology.

\section{CONCLUSION}

This proposed system discussed about the development of an android mobile application for outfit coordination. It is introduced to improve the people's awareness about the Indian laws and it also provides a simplified platform to communicate or interact with the lawyers. Ordinary peoples who are unaware of laws and procedures could use this App to find the appropriate procedures and the details about each and every laws. Legal documents and judgments (past case histories) from different courts are available for the lawyers which would be helpful for lawyers for practicing and this facility is implemented using data mining technology.

\section{REFERENCES}

[1]. Lefteris Zervakis, Christos Tryfonopoulos, Spiros Skiadopoulos and Manolis Koubarakis"Query Reorganisation Algorithms for Efficient Boolean Information Filtering" IEEE Transactions on Knowledge and Data Engineering ( Volume: 29 , Issue: 2 , Feb. 12017 )

[2]. D. Sangeetha, R. Kavyasri, S. Swetha, S. Vignesh "Information Retrieval System For Laws" 2016 Eighth International Conference on Advanced Computing (ICoAC), 19 June 2017

[3]. Shubhankar Mukherjee,Prof. Jyoti Prakash and Deepak Kumar "Android Application Development \& Its Security", International Journal of Computer Science and Mobile Computing (IJCSMC),Vol. 4, Issue. 3, March 2015, pg.714 - 719

[4]. siddhant singh "Android Application Development For Social Network", International Research Journal of Engineering and Technology (IRJET),Volume: 04 Issue: 12 | Dec-2017

[5]. P.Sathishkumar, P.Sabarinath, N.Sabarish and S.Swathi "Android Application For Law And Order Using Data Mining", International Journal of Advance Research and Innovative Ideas in Education (IJARIIE),Vol-2 Issue-4 2017

[6]. Abhinav Kathuria and Anu Gupta "Challenges in Android Application Development: A Case Study", International Journal of Computer Science and Mobile Computing (IJCSMC),Vol. 4, Issue. 5, May 2015, pg.294 - 299

[7]. Kavitha. R, Rupali Wagh , Remona Yacoop and Deeksha S“Design And Implementation Of On-Line Chatting Application Using Android”, International Journal Of Advanced Technology In Engineering And Science (IJATES), Volume No 03, Special Issue No. 01, April 2015

[8]. Shubham Pandey, K. Navin and G. Vadivu "Design of Cloud based Instant Messaging System on Android Smartphone using Internet", International Journal of Computer Applications (IJCA), Volume 89 - No 14, March 2014

[9]. Mamta, Hariom Tyagi and Abhishek Shukla " The Study of Electronic Payment Systems ", International Journal of Advanced Research in Computer Science and Software Engineering (IJARCSSE), Volume 6, Issue 7, July 2016

[10]. Tomi Dahlberg and Niina Mallat "Mobile Payment Market and Research - Past, Present and Future", Working Papers on Information Systems, http://sprouts.aisnet.org/6-48

[11]. Jianye Liu and Jiankun Yu “ Research on Development of Android Applications”,Fourth International Conference on Intelligent Networks and Intelligent Systems, 2011

[12]. Yongqiang He, Yanrong Shi and Aixiang He"Research on Online Payment Mode of E-commerce", IEEE International Conference on Software Engineering and Service Sciences, 19 August 2010

[13]. Gurpreet Singh Sambhy , Study of Mobile Payment Services in India [Online]. Available:http://www.divaportal.org/smash/get/diva2:696343/FULLTEXT01 